\title{
Effectiveness and Cost-Effectiveness of Dialectical Behaviour Therapy for Self-Harming Patients with Personality Disorder: A Pragmatic Randomised Controlled Trial
}

\author{
Stefan Priebe ${ }^{a} \quad$ Nyla Bhatti $^{b} \quad$ Kirsten Barnicot $^{a} \quad$ Stephen Bremner $^{a}$ \\ Amy Gagliab $^{\text {C Christina Katsakou }}{ }^{a, b}$ Iris Molosankwe ${ }^{c}$ Paul McCrone ${ }^{c}$ \\ Martin Zinkler ${ }^{\mathrm{a}}$ \\ ${ }^{a}$ Queen Mary University of London, ${ }^{\mathrm{b}}$ East London NHS Foundation Trust, and ${ }^{\mathrm{C}}$ Institute of Psychiatry, \\ King's College London, London, UK
}

\section{Key Words}

Dialectical behaviour therapy · Borderline personality

disorder $\cdot$ Self-harm $\cdot$ Cost-effectiveness

\begin{abstract}
Background: A primary goal of dialectical behaviour therapy (DBT) is to reduce self-harm, but findings from empirical studies are inconclusive. The aim of this study was to assess the effectiveness and cost-effectiveness of DBT in reducing self-harm in patients with personality disorder. Methods: Participants with a personality disorder and at least 5 days of self-harm in the previous year were randomised to receive 12 months of either DBT or treatment as usual (TAU). The primary outcome was the frequency of days with self-harm; secondary outcomes included borderline personality disorder symptoms, general psychiatric symptoms, subjective quality of life, and costs of care. Results: Forty patients each were randomised to DBT and TAU. In an intention-to-treat analysis, there was a statistically significant treatment by time interaction for self-harm (incidence rate ratio 0.91, 95\% $\mathrm{Cl} 0.89-0.92, \mathrm{p}<0.001$ ). For every 2 months spent in DBT, the risk of self-harm decreased by $9 \%$ relative to TAU. There was no evidence of differences on any secondary outcomes. The
\end{abstract}

economic analysis revealed a total cost of a mean of 5,685 GBP $(6,786$ EUR) in DBT compared to a mean of 3,754 GBP (4,481 EUR) in TAU, but the difference was not significant (95\% Cl -603 to 4,599 GBP). Forty-eight per cent of patients completed DBT. They had a greater reduction in self-harm compared to dropouts (incidence rate ratio $0.78,95 \% \mathrm{Cl}$ 0.76-0.80, $p<0.001)$. Conclusions: DBT can be effective in reducing self-harm in patients with personality disorder, possibly incurring higher total treatment costs. The effect is stronger in those who complete treatment. Future research should explore how to improve treatment adherence.

Copyright $\odot 2012$ S. Karger AG, Basel

\section{Background}

Dialectical behaviour therapy (DBT) was developed [1, 2] for the treatment of patients with borderline personality disorder (BPD), with a primary focus on self-harm reduction. Since its development, it has gained increasing popularity as an effective treatment for people with BPD who regularly self-harm $[3,4]$. In the UK, DBT is the only psychosocial treatment recommended by the National Institute for Health and Clinical Excellence as a treat-

\section{KARGER}

Fax +4161306 1234 E-Mail karger@karger.ch www.karger.com
(C) 2012 S. Karger AG, Basel

$0033-3190 / 12 / 0816-0356 \$ 38.00 / 0$

Accessible online at:

www.karger.com/pps
Stefan Priebe

Unit for Social and Community Psychiatry, Queen Mary University of London

Barts and the London School of Medicine and Dentistry

Newham Centre for Mental Health, London E13 8SP (UK)

E-Mail s.priebe@qmul.ac.uk 
ment for women with BPD who engage in recurrent selfharm [5]. Empirical support for DBT has been demonstrated in a number of randomised controlled trials (RCTs) comparing DBT with control conditions including treatment as usual (TAU), comprehensive validation therapy and treatment by experts [6-13]. A meta-analysis of 16 DBT studies found a moderate effect size for DBT on self-harm in patients with BPD. However, this was only when compared with TAU; when compared with other treatments specific to BPD, the effect size was small [14].

Results on the effectiveness of DBT for treating selfharm are mixed. Of 8 RCTs, 2 found significant effects of DBT on the reduction of self-harm or suicidal behaviours, respectively $[6,11]$ and a third showed that patients receiving DBT had a greater reduction in self-harm frequency over time than those receiving TAU [10]. The remaining 5 RCTs, however, found no significant effect of DBT on the frequency of self-harm [7-9, 12, 13]. Whilst early literature reviews painted a largely positive picture of the effects of DBT on self-harm reduction $[3,15]$, recent thinking has been more cautious, including suggestions that DBT has been taken up by health care systems in both the USA and the UK relatively quickly despite minimal evidence [13].

Given the inconsistent findings on self-harm for a therapy specifically developed to ameliorate this outcome, further investigation into the treatment effects is clearly needed. Investigating self-harm is of particular importance due to the prevalence of self-harm in society, estimated at between 4.6 and $6.6 \%$ in the UK [16], and the resulting costs incurred by the health system. A recent study demonstrated that patients who engaged in 5 or more episodes of self-harm had the highest level of resource costs in the 6-month period around an episode [17]. Patients with BPD, a diagnosis for which self-harming is a diagnostic criterion, have more frequent attendances in Accident and Emergency Departments and psychiatric hospitalisations [18] and make more use of outpatient mental health services compared to patients with other axis II disorders [19] or patients with common mental illness [20]. Consequently, the treatment of selfharm, especially in the context of borderline and other personality disorders, has been identified as a priority for the National Health Service (NHS) in the UK [5, 21]. Given these findings, assessing the cost-effectiveness of psychotherapy is clearly important [22]. Whilst DBT is recommended by the National Institute for Health and Clinical Excellence as the treatment of choice for women with personality disorder who self-harm, to date there has been no evidence assessing the cost-effectiveness, nor the overall effectiveness of DBT in specifically reducing selfharm within an NHS context.

We aimed to test: (1) the effectiveness and cost-effectiveness of 12 months of DBT as compared to TAU in reducing self-harm in patients with a personality disorder; (2) the impact of DBT versus TAU on other outcome measures including BPD symptoms, general psychiatric symptoms, and quality of life. We hypothesised that patients receiving DBT would over time experience a greater reduction in days with self-harm compared to patients in TAU.

\section{Method}

\section{Design}

A pragmatic RCT assessing the effectiveness of 12 months of DBT compared to 12 months of TAU in the reduction of self-harm in patients with personality disorder.

\section{Participants}

Inclusion criteria were 5 days or more with self-harm in the year prior to treatment, age 16 years or over, and a diagnosis of at least one personality disorder. Though the majority of patients referred to the service who frequently self-harm have a diagnosis of BPD (91\% [23]), there is no theoretical basis to exclude people without a diagnosis of BPD, therefore people with any personality disorder were included in the study. Exclusion criteria were severe learning difficulties that would interfere with the individual's ability to participate in DBT treatment, and an inability to read or write English. Eligibility criteria were deliberately kept lenient to reflect the pragmatic nature of the trial. Eligible participants were informed about the study and asked for consent to be contacted by a researcher. Recruitment took place between March 2008 and May 2010 from referrals to the DBT service in the London Borough of Newham. Referrals were accepted from different sources including primary, secondary and tertiary services.

\section{Interventions}

Patients randomised to DBT received 12 months of DBT delivered according to Linehan's $[1,2]$ treatment and skills training manuals. DBT is based on the principles of cognitive behavioural therapy with the inclusion of mindfulness, validation and supportive therapy techniques, and holds as its core the key dialectic of the acceptance of the individuals as they are with the acknowledgement of the need for change. It consists of weekly hour-long individual therapy sessions, a weekly 2-hour skills training group, and out-of-hours skills coaching over the telephone as needed. Treatment dropout was defined as missing 4 consecutive sessions of either the skills group, the individual sessions, or any combination of the two. To establish adherence to the DBT model, individual treatment sessions were audio recorded and $10 \%$ of the available recordings were assessed for adherence by a DBT therapist (A.G.) using a 63-item rating scale, providing an overall score ranging from 0 to 5 . A rating of 4.0 is regarded as reflecting good adherence. A further $10 \%$ of those assessments were rated by a second person to establish interrater reliability of the scores. Five 
group sessions were also rated for adherence to the DBT model using this scale.

Participants allocated to the TAU condition were referred back to the referrer and encouraged to engage in any kind of treatment other than DBT; this may have included treatment from psychotherapists, psychiatrists, community mental health teams, counsellors, general practitioners or user-run support groups, all of which were offered free of charge under the NHS. This condition therefore reflected as closely as possible the heterogeneous and multidisciplinary nature of the TAU services received by patients with BPD in the NHS. This is in accordance with the pragmatic nature of the trial in which we aimed to establish the real-world effectiveness of DBT relative to the real-world alternative treatments available.

\section{Baseline Measures}

The Structured Clinical Interview for DSM-IV [24] was used by all therapists prior to randomisation and at baseline to assess axis II disorders, including borderline personality disorder. Axis I disorders according to DSM-IV criteria were measured at baseline by a researcher using the Mini International Neuropsychiatric Interview [25]. Participants' age, ethnic background and gender were also recorded by the researcher at baseline.

\section{Outcomes}

Primary Outcome. The primary outcome was self-harm, measured in number of days over the 12-month period. Self-harm was defined as any act which (a) the individual performed with the intention to self-harm, and (b) caused tissue damage. Days of selfharm and type of deliberate self-harm were recorded in an interview on a structured form. This was assessed for the previous 12 months at the baseline assessment, and for the previous 2 months at each 2-month follow-up thereafter.

Secondary Outcomes. Borderline personality disorder symptoms were assessed at baseline and 12 months after randomisation using the Zanarini Rating Scale for Borderline Personality Disorder [26], a measure adapted from the 9 criteria for BPD according to the DSM-IV. The Zanarini Rating Scale of Borderline Personality Disorder is an interview-based measure on a Likert scale of 0-4 for each item. The total score ranges from 0 to 36 with higher scores reflecting greater severity of symptoms. Psychiatric symptoms were measured on the 24-item Brief Psychiatric Rating Scale [27], and the 53-item Brief Symptom Inventory [28]. On the Brief Symptom Inventory, symptoms are self-rated on Likert type scales. The sum scores ranges from 0 to 212, with higher scores indicating higher symptom levels. On the Brief Psychiatric Rating Scale, symptoms are observer-rated with total scores ranging from 24 to 168. For the Brief Psychiatric Rating Scale, interrater reliability between 5 researchers who independently rated symptoms was excellent [29] (intraclass correlation coefficient $=0.87$ ). Subjective quality of life was measured on the 12-item Manchester Short Assessment of Quality of Life [30]. Patients rate their satisfaction with life in general and different life domains on Likert type scales ranging from 1 (couldn't be worse) to 7 (couldn't be better). All secondary outcomes were assessed at baseline and at 12 months.

\section{Costs}

For the economic analysis, retrospective information pertaining to the participant's use of health and social care services was measured using a modified version of the Client Service Receipt Inventory [31], administered at baseline and every 2-month period subsequently. Service use included detailed information on the number of days spent in hospital, outpatient visits, contacts with general practitioners and other community-based health providers, details of prescribed medication, as well as lost work time for those in employment.

\section{Randomisation and Procedure}

After the baseline assessment, participants were randomly allocated to one of two groups: DBT or TAU. Randomisation was computer generated with a 1:1 allocation by an independent statistician, using 6 blocks of 12 randomly permuted treatment allocation sequences, with a final block of 8 . To have reliable patient reports on their behaviour with little distortion through memory bias, we assessed the primary outcome and costs every 2 months. The cost assessment included questions on service use which revealed the patients' allocation to the DBT or TAU group. Interviewers were therefore not masked to treatment allocation. However, the data analyst remained masked throughout the study period. Participants were offered 10 GBP for every research appointment attended.

\section{Statistical and Economic Analysis}

For the primary outcome number of self-harm days, a mixedeffects Poisson regression model was defined in order to use all of the available repeated measures data whilst accounting for the non-independence of repeated measures within the same individual. The interaction effect of allocation group and time was investigated for the outcome days with self-harm within each 2 -month period. Assuming missing covariate values were missing at random, maximum likelihood estimation ensured unbiased parameter estimates. In line with recommendations for interpreting treatment by time interactions in trials, the main effect of treatment was not included in the interaction model, as doing so made the model more robust against violating the assumption that the treatment effects are linear with time [32]. Additionally, the average number of days with self-harm in the past 2 months at baseline was calculated and included in the outcome as the randomisation procedure negated the need to estimate a difference in baseline self-harm. We also conducted a sensitivity analysis with last observation carried forward.

For each of the secondary analyses, linear regression models were fitted. The main effect of allocation group on outcome was estimated, adjusting for baseline value of the outcome.

Following a previous audit in the same service where a treatment dropout rate of $63 \%$ was observed [23], a high treatment dropout rate in the DBT arm was expected. In an a priori planned subgroup analysis comparing the primary outcome in completers and dropouts we used the same model as for the primary outcome in the total sample and computed the completer versus dropout by time interaction on self-harm, with baseline scores included in the outcome.

For the economic analyses, a comprehensive approach was taken with services including primary and secondary care and social care. Service costs were calculated by combining these data with appropriate national unit costs [33]. Lost work days were measured and valued using average wage rates. Service costs and total costs (i.e. including lost employment) were compared using a bootstrapped regression model given the expected skewness of 
Fig. 1. CONSORT diagram of flow of participants through the study.

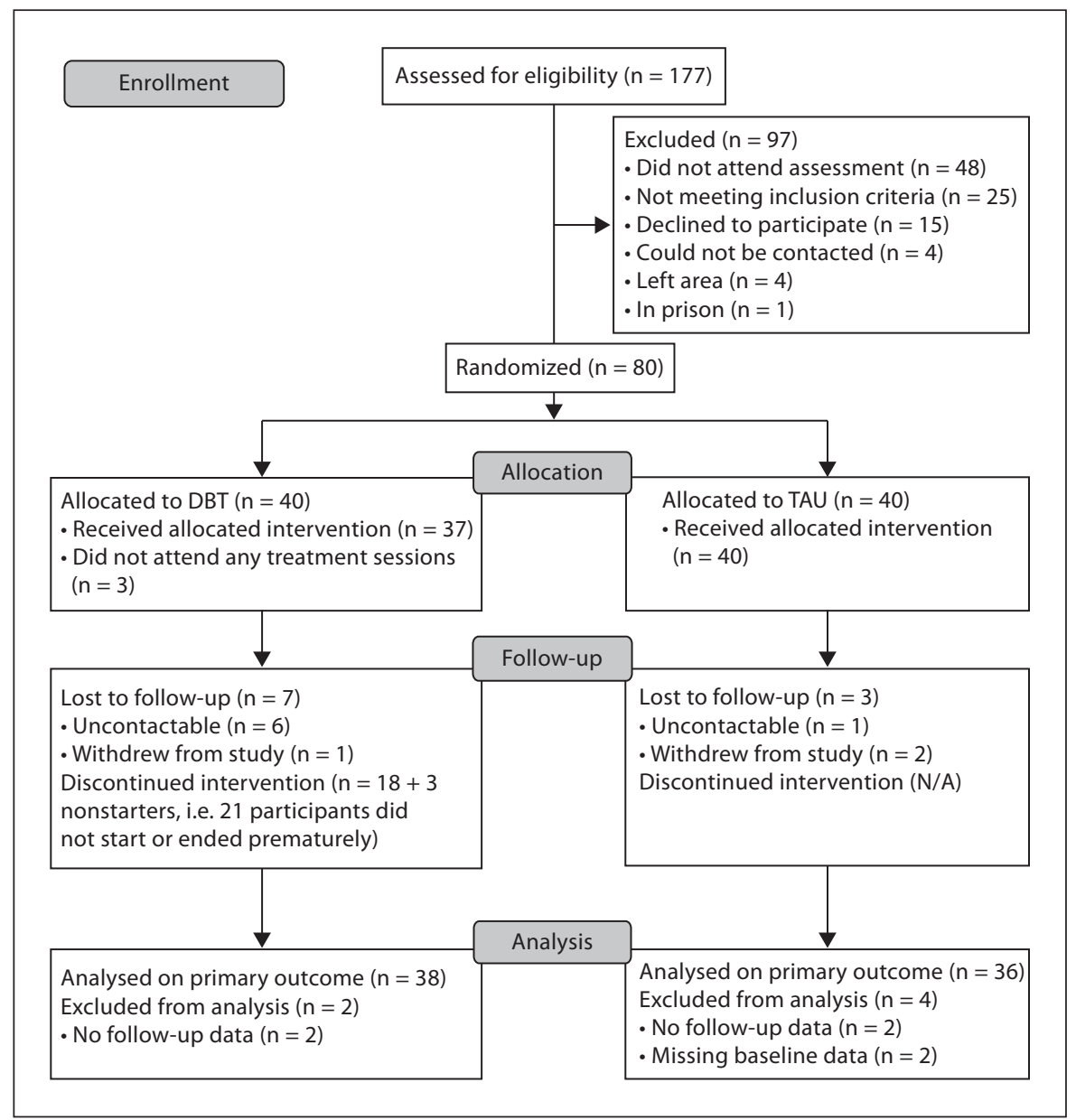

cost data. Cost-effectiveness was assessed by producing an incremental cost-effectiveness ratio which indicates the cost per percentage point difference in self-harm rates.

\section{Results}

\section{Participant Characteristics}

Figure 1 shows the flow of participants through the study. During the recruitment period, 177 patients were referred to the service, of which 80 were eligible for and agreed to participate in the study. Recruitment to the study ceased once this figure was achieved, and 40 were randomised to each condition. Of the 40 randomised to receive DBT, 3 people did not start treatment due to various reasons and a further 18 patients did not complete the 1-year treatment (fig. 1). Demographic and clinical characteristics at baseline are summarised in table 1 (see also online suppl. table 1; for all online suppl. material, see www.karger.com/doi/10.1159/000338897).

RCT of DBT for Personality Disorder with Self-Harm

\section{Interventions}

A mean score of 4.1 (range 3.6-4.5) on the adherence rating scale demonstrated good adherence of the individual sessions to the DBT model. The two raters rated all sessions within a margin of 0.3 -point difference on the adherence scale. Skills teaching groups were also measured in the same way and were deemed to be adherent. Of the 18 patients allocated to DBT who started treatment but did not complete, seven ceased treatment in the first 3 months, three between 4 and 6 months, six between 7 and 9 months, and two in the last 3 months of treatment. Their mean length of stay in treatment was 5.7 months $(\mathrm{SD}=3.2)$.

Participants allocated to TAU received some form of mental health treatment from a range of services, summarised in online supplementary table 2.

Seven participants received individual therapy at any one time across the study period, including cognitive behavioural therapy, schema-focused therapy, psychody-

Psychother Psychosom 2012;81:356-365 
Table 1. Socio-demographic and clinical characteristics of patients in DBT and TAU at baseline

\begin{tabular}{|c|c|c|c|c|c|c|}
\hline \multirow[b]{3}{*}{ Age, years } & \multicolumn{2}{|c|}{ DBT } & \multicolumn{2}{|c|}{ TAU } & \multicolumn{2}{|c|}{ Total } \\
\hline & \multicolumn{2}{|l|}{$\mathrm{n}$} & \multicolumn{2}{|l|}{$\mathrm{n}$} & \multicolumn{2}{|l|}{$\mathrm{n}$} \\
\hline & 40 & $33.0 \pm 10.7$ & 40 & $31.3 \pm 11.0$ & 80 & $32.2 \pm 10.8$ \\
\hline Female & 40 & $35(87.5 \%)$ & 40 & $35(87.5 \%)$ & 80 & $70(87.5 \%)$ \\
\hline Ethnicity & 40 & & 40 & & 80 & \\
\hline White & & $26(65 \%)$ & & $20(50 \%)$ & & $46(57.5 \%)$ \\
\hline Black & & $4(10 \%)$ & & $8(20 \%)$ & & $12(15 \%)$ \\
\hline Asian & & $8(20 \%)$ & & $9(22.5 \%)$ & & $17(21.3 \%)$ \\
\hline Mixed/other & & $2(5 \%)$ & & $3(7.5 \%)$ & & $5(6.3 \%)$ \\
\hline Employment & 40 & & 40 & & 80 & \\
\hline None & & $22(55 \%)$ & & $21(52.5 \%)$ & & $43(53.8 \%)$ \\
\hline Voluntary/protected/sheltered work & & $4(10 \%)$ & & $4(10 \%)$ & & $8(10 \%)$ \\
\hline Regular employment & & $14(35 \%)$ & & $15(37.5 \%)$ & & $29(36.3 \%)$ \\
\hline Accommodation & 40 & & 40 & & 80 & \\
\hline Homeless or $24 \mathrm{~h}$ supervised & & $2(5 \%)$ & & $4(10 \%)$ & & $6(7.5 \%)$ \\
\hline Sheltered or supported accommodation & & $4(10 \%)$ & & $2(5 \%)$ & & $6(7.5 \%)$ \\
\hline Independent accommodation & & $34(85 \%)$ & & $34(85 \%)$ & & $68(85 \%)$ \\
\hline Self-harm days in past 2 months (averaged) & 40 & $14.7 \pm 20.3$ & 38 & $13.0 \pm 16.3$ & 78 & $13.9 \pm 18.4$ \\
\hline BPD symptom severity (scale range $0-36$ ) & 39 & $17.9 \pm 6.8$ & 40 & $18.4 \pm 7.6$ & 79 & $18.2 \pm 7.2$ \\
\hline Number of suicide attempts in past 12 months & 40 & $1.8 \pm 2.5$ & 39 & $2.6 \pm 8.2$ & 79 & $2.2 \pm 6.0$ \\
\hline Self-reported general psychiatric symptoms (BSI) & & & & & & \\
\hline (scale range $0-212$ ) & 40 & $122 \pm 41.2$ & 39 & $134 \pm 39.3$ & 79 & $127.5 \pm 40.5$ \\
\hline Observer-rated general psychiatric symptoms (BPRS) & & & & & & \\
\hline (scale range 24-168) & 39 & $50 \pm 5.6$ & 40 & $52.8 \pm 9.9$ & 79 & $51.9 \pm 8.7$ \\
\hline Quality of life (MANSA) (scale range 1-7) & 39 & $3.3 \pm 1.0$ & 40 & $3.2 \pm 3.3$ & 79 & $3.2 \pm 0.9$ \\
\hline Number of axis I disorders & 28 & $7.5 \pm 2.3$ & 35 & $8.5 \pm 3.2$ & 63 & $8.0 \pm 3.1$ \\
\hline Number of axis II disorders & 40 & $3.4 \pm 1.4$ & 40 & $3.5 \pm 1.8$ & 80 & $3.5 \pm 1.6$ \\
\hline
\end{tabular}

BSI = Brief Symptom Inventory; BPRS = Brief Psychiatric Rating Scale; MANSA = Manchester Short Assessment of Quality of Life.

namic therapy and multimodal therapy for an average of 5 months. Twelve participants had outpatient contact with a psychiatrist for an average of 8 months, and 7 participants had access to specialist outpatient mental health services for an average of 9 months. A total of 8 participants, or $20 \%$, received care from their general practitioner only across the 12 -month TAU period.

\section{Primary Outcome: Self-Harm}

Figure 2 shows the average number of days with selfharm per 2-month period for patients allocated to DBT and TAU.

Patients allocated to DBT showed a greater reduction of days with self-harm over time. There was a statistically significant interaction between treatment condition and time (incidence rate ratio $0.91 ; \mathrm{p}<0.001$ ). The interaction term demonstrated that the incidence rate of selfharm per 2-month period decreased by an additional $9 \%$ in the DBT group compared to the TAU group. Thus, for every 2 months in DBT, the risk of self-harm decreased relative to TAU by an additional $9 \%$. The $95 \%$ confidence interval for this additional reduction ranged from 8 to $11 \%$. The sensitivity analysis with last observation carried forward showed a very similar result (incidence risk ratio 0.91; $\mathrm{p}<0.001 ; 95 \%$ CI .89-.92)

Figure 3 shows the frequency of days with self-harm in patients who completed DBT, those who did not complete and the group receiving TAU. Patients who completed DBT started with a higher frequency of days with self-harm. Over time, they showed a greater reduction and after 12 months they had a lower frequency of selfharm as compared to those who did not complete DBT. In a subgroup analysis comparing changes in self-harm between treatment completers $(\mathrm{n}=19)$ and non-completers $(\mathrm{n}=21)$, a statistically significant treatment completion (vs. non-completion) by time interaction was observed (incidence rate ratio $0.78 ; \mathrm{p}<0.001$ ), indicating an additional $22 \%$ decrease in the incidence of self- 


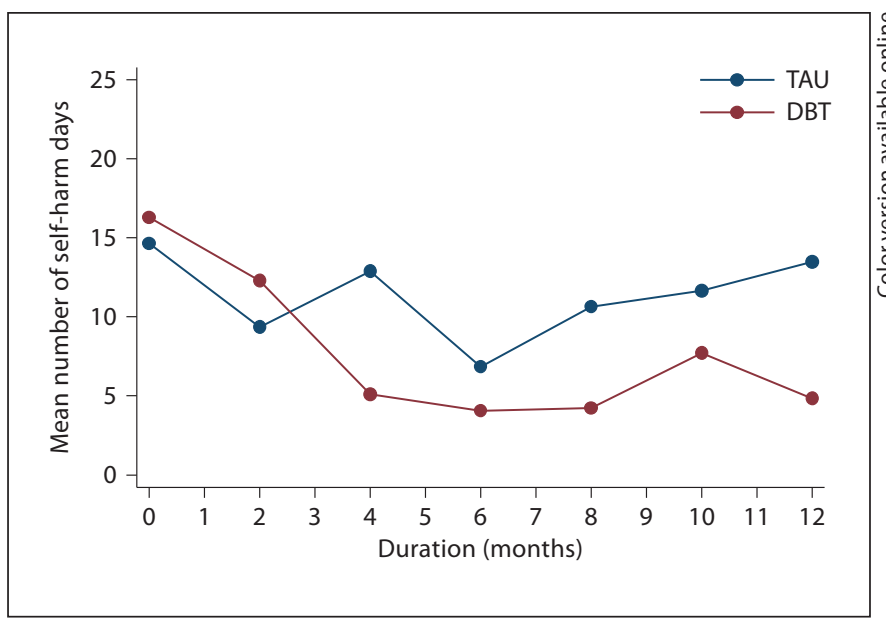

Fig. 2. Change in days with self-harm over time in patients in DBT and TAU.

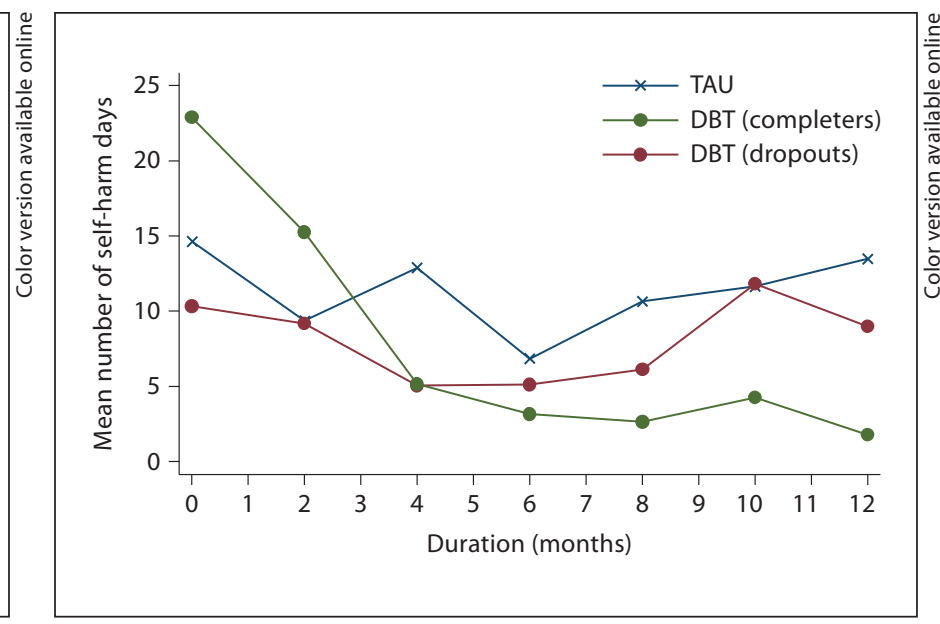

Fig. 3. Changes in days with self-harm over time in patients receiving TAU, in patients completing DBT and in patients dropping out of DBT.

Table 2. Scores of the Zanarini Rating Scale of Borderline Personality Disorder (ZAN-BPD), the Brief Psychiatric Symptom Inventory (BSI), the Brief Psychiatric Rating Scale (BPRS), and Manchester Short Assessment of Quality of Life (MANSA) for patients in DBT and TAU at baseline and at 12 months

\begin{tabular}{|c|c|c|c|c|c|c|c|c|}
\hline \multirow[t]{2}{*}{ Outcome } & & \multicolumn{2}{|c|}{$\mathrm{DBT}$} & \multicolumn{2}{|c|}{ TAU } & \multirow{2}{*}{$\begin{array}{l}\text { Effect } \\
\text { estimate }\end{array}$} & \multirow[t]{2}{*}{$95 \% \mathrm{CI}$} & \multirow[t]{2}{*}{$\mathrm{p}$ value } \\
\hline & & $\mathrm{n}$ & mean $(\mathrm{SD})$ & $\mathrm{n}$ & mean $(\mathrm{SD})$ & & & \\
\hline \multirow[t]{2}{*}{ ZAN-BPD } & baseline & 40 & $17.9(6.8)$ & 39 & $18.4(7.6)$ & \multirow[t]{2}{*}{$\beta=-2.4$} & \multirow[t]{2}{*}{-5.7 to 1.0} & \multirow[t]{2}{*}{0.16} \\
\hline & month 12 & 33 & $13.1(6.9)$ & 37 & $15.9(7.5)$ & & & \\
\hline \multirow[t]{2}{*}{ BSI } & baseline & 35 & $122(41.2)$ & 36 & $134(39.3)$ & \multirow[t]{2}{*}{$\beta=-3.2$} & \multirow[t]{2}{*}{-25.8 to 19.3} & \multirow[t]{2}{*}{0.77} \\
\hline & month 12 & 29 & $100.6(57.1)$ & 31 & $116(52.3)$ & & & \\
\hline \multirow[t]{2}{*}{ BPRS } & baseline & 39 & $50(5.6)$ & 33 & $52.8(9.9)$ & \multirow[t]{2}{*}{$\beta=-1.3$} & \multirow[t]{2}{*}{-4.4 to 1.7} & \multirow[t]{2}{*}{0.38} \\
\hline & month 12 & 40 & $48(11.7)$ & 34 & $51(10.9)$ & & & \\
\hline \multirow{2}{*}{$\begin{array}{l}\text { Quality of life (MANSA) } \\
\text { (scale range 1-7) }\end{array}$} & baseline & 39 & $3.3(0.8)$ & 40 & $3.2(0.9)$ & \multirow[t]{2}{*}{$\beta=0.3$} & \multirow[t]{2}{*}{-0.1 to 0.8} & \multirow[t]{2}{*}{0.10} \\
\hline & month 12 & 31 & $3.7(1.0)$ & 36 & $3.4(1.0)$ & & & \\
\hline
\end{tabular}

harm for every additional 2 months completers spent in DBT compared to non-completers. The $95 \%$ confidence interval for the additional reduction in treatment completers versus non-completers ranged from 20 to $24 \%$.

\section{Secondary Outcomes}

Secondary outcomes are summarised in table 2. In separate mixed model analyses, there was no significant effect of DBT on BPD symptoms, observer-rated and selfreported psychiatric symptom severity, or subjective quality of life at month 12 .

RCT of DBT for Personality Disorder with Self-Harm
Costs and Cost-Effectiveness

Service use and costs are shown in online supplementary table 3 . The highest cost item for the DBT patients was the DBT intervention, whilst inpatient costs were high for both DBT and TAU patients. Total service costs were higher for the DBT group (mean 5,685 \pm 6,431 GBP in DBT compared to 3,754 $\pm 6,045 \mathrm{GBP}$ in TAU), but the difference was not statistically significant (95\% CI -603 to $4,599 \mathrm{GBP})$. With the inclusion of lost employment costs the DBT group remained more costly, but again without the differences being statistically significant ( $95 \%$ CI $-1,299$ to 5,110 GBP). The average service cost difference per 2-month period was 322 GBP. Dividing 
this by the extra decrease in self-harm (9\%) indicates that it costs 36 GBP (approx. 41 EUR) to achieve a one percentage point reduction in the incidence of self-harm as a result of using DBT.

\section{Discussion}

\section{Main Findings}

This pragmatic trial assessed the effectiveness and cost-effectiveness of DBT compared with TAU in reducing self-harm for patients with personality disorder and yielded 3 main findings. Firstly, in an intention-to-treat analysis, the risk of self-harm in DBT significantly reduced by an additional $9 \%$ per 2 -month period relative to TAU, but DBT had no statistically significant effect on any other outcomes. Secondly, the costs to achieve a one percentage point reduction in self-harm with DBT per 2 -month period was $36 \mathrm{GBP}$. Finally, only $48 \%$ of patients allocated to the DBT group completed the envisaged 1-year treatment period, and the reduction of self-harm was substantially greater for those who completed the intervention than for those who did not.

\section{Strengths}

Self-harm was assessed in this study at more frequent time intervals (every 2 months) than in other RCTs of DBT. In retrospective recall, frequently occurring events over periods of 6 months are often underreported [34], and the regular data collection every 2 months allowed a more reliable and accurate measure of self-harm over a year-long course of DBT compared to when patients have to recall frequency of self-harm over longer periods of time [6-13]. Although more than $50 \%$ of patients in DBT did not start DBT or dropped out of treatment, outcome data were still obtained for most of the participants. DBT therapists were found to be adherent to the DBT framework ensuring that the intervention delivered was DBT as prescribed by the treatment manual. In accordance with the pragmatic nature of the trial, the selection criteria and TAU condition were designed to reflect the realworld equivalents; this is in contrast to previous RCTs of DBT which employed stringent selection criteria, commonly excluding males [6-11] and those with a substance use disorder $[6,8,12,13]$, and sometimes had unrepresentative and costly control conditions. Finally, this is the first RCT to assess the cost-effectiveness of DBT in the NHS in the UK, where all care is free at the point of entry and patients in TAU as well as dropouts of DBT have a range of alternative treatment options.

\section{Limitations}

Whilst we aimed to assess each participant of the study at 2-month intervals, there were some missing data. For the primary outcome, the total number of days with selfharm were counted which may have underestimated the frequency of self-harm acts (some patients may have repeatedly self-harmed on the same day) and does not take account of the severity of those acts. Due to the nature of the questions researchers asked regarding service use, coupled with the frequency of contact between researchers and participants (every 2 months) it was impractical to blind researchers to allocation; this may have biased the way in which the interviews were conducted. Additionally, referring those allocated to TAU back to their original referral sources following randomisation may have had a negative impact on participants, which may have augmented any negative outcomes observed in this group. Finally the small sample size may have provided insufficient statistical power to establish a significant difference in costs between DBT and TAU.

\section{Comparison with Previous Research Findings}

This study demonstrated a greater decrease in selfharm over time for patients receiving DBT as compared to those in TAU. This was statistically significant, and the $95 \%$ confidence interval indicated only a small margin of measurement error between 8 and $11 \%$. The finding is congruous with two previous RCTs in which DBT was associated with a favourable reduction of self-harm over time $[8,10]$, but in contrast to all other RCTs, which have failed to demonstrate an effect of DBT on self-harm $[6,7$, $9,11-13]$.

The control conditions adopted in these studies have ranged from individual psychotherapy [6-8], therapy tailored specifically for the group studied (comprehensive validation therapy for substance abuse [9]; community treatment by experts [11]), clinical management in the community $[10,12]$ or standard NHS care [13]. The pragmatic nature of our trial required a less stringent control condition, and this might have enhanced the relative effect of DBT on self-harm. However, the pragmatic nature of the trial with a less stringent control condition reflects the decision that commissioners of services have to make in the context of many health care systems: the question is whether to commission and fund DBT as compared to TAU (which in Western Europe commonly includes a number of alternative options) and not as compared to another well-defined treatment.

The treatment completion rate of $48 \%$ in the current study is in contrast to a meta-analysis of 14 studies of pa- 
tients with BPD undergoing a 12-month or longer course of DBT outside the UK, showing an average completion rate of $70 \%$ [35]. Similarly, the average treatment completion rate in 7 RCTs assessing the effect of DBT on selfharm outside the UK was 67\% [6-12]. Though 1 of the 3 primary objectives of DBT is to increase treatment retention [6], the comparatively high dropout rate in this study suggests that it is a struggle to achieve high completion rates of DBT in the NHS context. One reason may be the sample characteristics of the population studied; the sample was intended to be a reflection of a typical clinical population, so that the selection criteria were wide and inclusive. This is in contrast to other studies where only women were included and people with a number of comorbid diagnoses were excluded. Indeed, patients with BPD and a substance use disorder have demonstrably lower treatment retention rates [36] than patients with BPD without a substance use disorder. Thus the wide inclusion criteria in the current study may have contributed to the high dropout. Additionally, in many studies with a lower attrition rate patients have only limited access to other free health services outside of the study. One may speculate that participants may be more inclined to stay in treatment in places such as the USA, as dropping out might lead to costly health care alternatives, whilst in the $\mathrm{UK}$, the NHS provides free health care, including various psychological therapies, thus decreasing the incentive to stay in treatment.

\section{Clinical Implications}

The findings suggest that DBT may indeed reduce selfharm in patients with personality disorder. Whilst the difference between the DBT and TAU group in the intention-to-treat analysis of a $9 \%$ greater reduction in selfharm every 2 months may be seen as limited, the gain of those who completed treatment with a $22 \%$ greater reduction every 2 months as compared to dropouts is substantial. The clinical significance of this reduction may be seen as justifying the additional costs in the DBT group.

Treatment adherence, however, is a major problem. There may be a need to adjust the often strict adherence rules of DBT, the time commitment of a minimum of $3 \mathrm{~h}$ per week, or other aspects of the manualised approach to increase completion rates. At the same time, one may speculate as to whether such greater flexibility would compromise the effectiveness of DBT and that the challenge is rather to identify potential dropouts early and develop alternative treatment methods for them. Further research should explore the vulnerability factors associated with premature dropout prior to and during therapy.
A systematic review of treatment attrition in BPD patients undergoing psychotherapy [35] highlighted factors such as poor therapeutic alliance, low motivation to change and high impulsivity as predictors of therapy attrition. Recent neuropsychological research has also demonstrated that lower executive control and visual memory performance predict dropout [37]. Measures of such factors could be used for improving completion rates in DBT as well as in other psychotherapies which have been demonstrated to improve outcomes in the BPD population, such as transference-focused therapy [38], schema-focused therapy [39], STEPPS [40], mentalisation-based therapy [41], and narrative exposure therapy [42]. Additionally, research on the effectiveness of different levels of care (inpatient, day and outpatient treatments) available to personality disorder patients could shed light on treatment adherence across different modalities [43].

Whilst the current study found a significant effect of DBT on the primary outcome of self-harm, there was no significant impact on any of the secondary outcomes. It is unlikely that this was due to the limited sample size, as the observed differences between the two groups - although slightly more favourable for the DBT group were small and unlikely to be of much clinical significance even if they had been found to be statistically significant in a trial with a much larger sample.

One explanation may be that therapists focus on highrisk behaviour, i.e. self-harm. The remaining clinical symptoms and quality of life are then deemed less of a priority, in line with the DBT treatment targets hierarchy [44]. One may conclude that for self-harming patients with personality disorder DBT should be offered primarily as a means to reduce the high-risk behaviour rather than as an effective treatment for other clinical symptoms no matter whether they are specific to the diagnosis of BPD or more general.

\section{Future Directions}

The costs for DBT in the intervention group were partly, but not fully, offset by higher costs for other interventions in the control group. Although the cost differences between the DBT and the TAU group were not statistically significant with highly variable costs in both groups, the effect of DBT in reducing self-harm is likely to have a cost. The findings of this study estimated a modest investment for the achieved improvement, but further studies in larger samples and cost assessments outside the restricted conditions of research studies may be required to establish the additional costs of implementing DBT 
across the NHS and other health care systems. Moreover, whether or not the clinical and economical gains in the DBT group are maintained remains to be investigated using longitudinal methods.

Whilst DBT appears effective in reducing self-harm, the processes of achieving this remain unclear. Future research should investigate which specific and non-specific processes facilitate the reduction of self-harm in DBT to improve the approach of DBT and possibly utilise similar mechanisms in other and less formal models of treatment.

\section{Acknowledgements}

This paper presents independent research funded by the National Institute for Health Research (NIHR) under its Research for Patient Benefit Programme (grant reference No. PB-
PG-0906-10540). All authors were funded by this grant with the exception of K.B., whose contribution was funded by the NIHR Doctoral Research Fellowship scheme. The views expressed are those of the authors and not necessarily those of the NHS, the NIHR or the Department of Health. We are grateful to Prof. Peter Tyrer (Imperial College, London) who advised the team, the steering group with academics, clinicians and service users, the Newham DBT service lead Tim Mold and all the DBT therapists who agreed to participate in the study. Finally, we would like to acknowledge Matina Marougka, Naomi Fearns and Mark Savill who helped with data collection, and are grateful to all interviewed patients who agreed to participate.

\section{Disclosure Statement}

The authors declare that they have no conflicts of interest.

\section{References}

1 Linehan MM: Cognitive-Behavioral Treatment of Borderline Personality Disorder. New York, Guildford Press, 1993.

2 Linehan MM: Skills Training Manual for Treating Borderline Personality Disorder. New York, Guildford Press, 1993.

-3 Swenson CR, Torrey WC, Koerner K: Implementing dialectical behavior therapy. Psychiatr Serv 2002;53:171-178.

4 Feigenbaum J: Dialectical-behavior therapy: an increasing evidence base. J Ment Health 2007; 16:51-68

5 National Institute for Health and Clinical Excellence: Borderline Personality Disorder: Treatment and Management. London, $\mathrm{Na}-$ tional Institute for Health and Clinical Excellence, 2009.

-6 Linehan MM, Armstrong HE, Suarez A, Allmon D, Heard HL: Cognitive-behavioral treatment of chronically parasuicidal borderline patients. Arch Gen Psychiatry 1991; 48:1060-1064.

7 Linehan MM, Schmidt H, Dimeff LA, Craft JC, Kanter J, Comtois KA: Dialectical behavior therapy for patients with borderline personality disorder and drug-dependence. Am J Addict 1999;8:279-292.

$\checkmark 8$ Koons CR, Robins CJ, Tweed JL, Lynch TR, Gonzalez AM, Morse JQ, Bishop GK, Butterfield MI, Bastian LA: Efficacy of dialectical behavior therapy in women veterans with borderline personality disorder. Behav Ther 2001:32:371-390.

-9 Linehan MM, Dimeff LA, Reynolds SK, Comtois KA, Welch SS, Heagerty P, Kivlahan DR: Dialectical behavior therapy versus comprehensive validation therapy plus 12 step for the treatment of opioid dependent women meeting criteria for borderline personality disorder. Drug Alcohol Depend 2002;67:13-26.

10 Verheul R, Van Den Bosch LMC, Koeter MWJ, Ridder MAJ, Stijnen T, Van Den Brink W: Dialectical behaviour therapy for women with borderline personality disorder. $\mathrm{Br} \mathrm{J}$ Psychiatry 2003;182:135-140.

11 Linehan MM, Comtois KA, Murray AM Brown MZ, Gallop RJ, Heard HL, Korslund KE, Tutek DA, Reynolds SK, Lindenboim N: Two-year randomised controlled trial and follow-up of dialectical behavior therapy vs therapy by experts for suicidal behaviors and borderline personality disorder. Arch Gen Psychiatry 2006;63:757-767.

12 McMain SF, Links PS, Gnam WH, Guimond T, Cardish RJ, Korman L, Streiner DL: A randomized trial of dialectical behavior therapy versus general psychiatric management for borderline personality disorder. Am J Psychiatry 2009;166:1365-1374.

13 Feigenbaum JD, Fonagy P, Pilling S, Jones A, Wildgoose A, Bebbington PE: A real-world study of the effectiveness of DBT in the UK National Health Service. Br J Clin Psychol 2012;51:121-141.

14 Kliem S, Kroger C, Kosfelder J: Dialectical behavior therapy for borderline personality disorder: a meta-analysis using mixed-effects modelling. J Consult Clin Psychol 2010; 78:936-951.

15 Koerner K, Dimeff LA: Further data on dialectical behavior therapy. Clin Psychol 2000; 7:104-112.

16 Meltzer H, Lader D, Corbin T, Singleton N, Jenkins R, Brugha T: Non-Fatal Suicidal Behaviour among Adults Aged 16 to 74 in Great
Britain. London, The Stationery Office, 2002.

17 Sinclair JM, Gray A, Rivero-Aria O, Saunders KE, Hawton K: Healthcare and social services resource use and costs of self-harm patients. Soc Psychiatry Psychiatr Epidemiol 2011;46:263-271.

-18 Bender DS, Dolan RT, Skodol AE, Sanislow CA, Dyck IR, McGlashan TH, Shea MT, Zanarini MC, Oldham JM, Gunderson JG: Treatment utilization by patients with personality disorders. Am J Psychiatry 2001; 158:295-302.

19 Hörz S, Zanarini MC, Frankenburg FR, Reich DB, Fitzmaurice G: Ten-year use of mental health services by patients with borderline personality disorder and with other axis II disorders. Psychiatr Serv 2010;61: 612-616.

20 Ansell EB, Sanislow CA, McGlashan TH, Grilo CM: Psychosocial impairment and treatment utilization by patients with borderline personality disorders, mood and anxiety disorders, and a healthy comparison group. Comp Psychiatry 2007;48:329-336.

21 National Institute for Mental Health in England: Personality Disorder: No Longer a Diagnosis of Exclusion. London, National Institute for Mental Health in England, 2003.

22 Davidson KM, Tyrer P, Norrie J, Palmer SJ, Tyrer H: Cognitive therapy v usual treatment for borderline personality disorder: prospective 6-year follow-up. Br J Psychiatry 2010; 197:456-462.

23 Zinkler M, Gaglia A, Arokiadass SMR, Farhy E: Dialectical behaviour treatment: implementation and outcomes. Psychiatr Bull 2007;31:249-252. 
24 First MB, Gibbon M, Spitzer RL, Williams JBW, Benjamin LS: Structured Clinical Interview for DSM-IV Axis II Personality Disorders (SCID-II). Washington, American Psychiatric Press Inc, 1997.

-25 Sheehan DV, Lecrubier Y, Sheehan KH, Amorim P, Janavs J, Weiller E, Hergueta T, Baker R, Dunbar GCL: The Mini International Neuropsychiatric Interview (MINI): the development and validation of a structured diagnostic psychiatric interview for DSM-IV and ICD-10. J Clin Psychiatry 1998; 59:22-33.

26 Zanarini MC: Zanarini rating scale for borderline personality disorder (ZAN-BPD): a continuous measure of DSM-IV borderline psychopathology. J Pers Disord 2003;17:233242 .

27 Ventura J, Green MF, Shaner A, Liberman RP: Training and quality assurance with the Brief Psychiatric Rating Scale: 'the drift busters'. Int J Methods Psychiatr Res 1993;3:221224.

28 Derogatis LR: Brief Psychiatric Inventory. Baltimore, Clinical Psychometric Research, 1975.

-29 Burdock E, Fleiss JL, Hardesty AS: A new view of interobserver agreement. Pers Psychol 1963;16:373e84.

-30 Priebe S, Huxley P, Knight S, Evans S: Application and results of the Manchester short assessment quality of life (MANSA). Int J Soc Psychiatry 1999;45:7-12.
31 Beecham J, Knapp M: Costing psychiatric interventions; in Thornicroft G (ed): Measuring Mental Health Needs, ed 2. London, Gaskell, 2001.

32 Cook TD, DeMets DL (eds): Introduction to Statistical Methods for Clinical Trials. Boca Raton, Taylor \& Francis Group, 2008.

33 Curtis L: Unit Costs of Health and Social Care. Canterbury, PSSRU, 2010.

34 Jobe JB, White AA, Kelley CL: Recall strategies and memory of health care visits. Milbank Q 1990;68:171-189.

-35 Barnicot K, Katsakou C, Marougka S, Priebe S: Treatment completion in psychotherapy for borderline personality disorder - A systematic review and meta-analysis. Acta Psychiatr Scand 2011;123:327-338.

36 Bornalova MA, Daughters SB: How does dialectical behavior therapy facilitate treatment retention among individuals with comorbid borderline personality disorder and substance use disorders? Clin Psychol Rev 2007; 27:923-943

37 Fertuck EA, Keilp J, Song I, Morris MC, Wilson ST, Brodsky BS, Stanley B: Higher executive control and visual memory performance predict treatment completion in borderline personality disorder. Psychother Psychosom 2012;81:38-43.

38 Clarkin JF, Levy KN, Lezenweger MF, Kernberg OF: Evaluating three treatments for borderline personality disorder: a multiwave study. Am J Psychol 2007;164:922-928.
39 Giesen-Bloo J, van Dyck R, Spinhoven P, van Tilburg W, Dirksen C, van Asselt T, Kremers I, Nadort M, Arntz A: Outpatient psychotherapy for borderline personality disorder. Randomized trial of schema-focused therapy vs transference-focused therapy. Psychother Psychosom 2006;63:649-659.

40 Bos EH, van Wel EB, Appelo MT, Verbraak MJPM: Effectiveness of Systems Training for Emotional Predictability and Problem Solving (STEPPS) for borderline personality problems in a 'real-world' sample: moderation by diagnosis or severity? Psychother Psychosom 2011;80:173-181.

41 Bateman A, Fonagy P: Randomized controlled trial of outpatient mentalizationbased treatment versus structured clinical management for borderline personality disorder. Am J Psychiatry 2009;166:1355-1364.

42 Pabst A, Schauer M, Bernhardt K, Ruf M, Goder R, Rosentraeger R, Elbert T, Aldenhoff J, Seeck-Hirschner M: Treatment of patients with borderline personality disorder and comorbid posttraumatic stress disorder using narrative exposure therapy: a feasibility study. Psychother Psychosom 2012;81: 61-63.

43 Ogrodniczuk JS: New directions in treatment research for personality disorders: effectiveness of different levels of care. Psychother Psychosom 2011;80:65-69.

44 Robins CJ, Chapman AL: Dialectical behavior therapy: current status, recent developments, and future directions. J Pers Disord 2004;18:73-89. 